\title{
Macroscopic quantum vacuum and microscopic gravitation
}

\author{
Roberto Onofrio ${ }^{1,2,3}$ \\ ${ }^{1}$ Dipartimento di Fisica "Galileo Galilei", Università di Padova, Padova 35131, Italy \\ ${ }^{2}$ Department of Physics and Astronomy, Dartmouth College, Hanover, NH 03766, USA \\ ${ }^{3}$ ITAMP, Harvard-Smithsonian Center for Astrophysics, Cambridge, MA 02138, USA
}

\begin{abstract}
Macroscopic quantum vacuum and modern theories of gravitation share the strong interplay between geometry and physical phenomena. We review selected issues related to the accuracy of the measurement of Casimir forces with particular emphasis on the implications for the search of non-Newtonian gravitational forces in the micrometer range. We then discuss the interplay of the Higgs particle with gravitation, arguing that spectroscopic shifts in atomic transitions due to the modifications of the vacuum expectation value of the Higgs field in regions with strong curvature of space-time may be of conceptual and observational relevance.
\end{abstract}

PACS numbers:

\section{INTRODUCTION}

Casimir forces have been extensively studied in the last decade in a variety of experimental configurations [1, 2]. At the level of demonstrating the existence of this peculiar manifestation of quantum vacuum at the macroscopic level, the situation seems rather settled. A demonstration of an effect which is expected since it is based on the prevailing theory, i.e. renormalized quantum electrodynamics, without predictions originating from alternative approaches [3], seems rather uneventful and does not necessarily require an in-depth analysis of the data. Debate, however, is still going on to assess the precision and the accuracy of the measurements, since this has an impact on the potential discovery of new forces - predicted by a variety of models - with coupling comparable or larger than gravitation, and characterized by an interaction range at or below the micrometer scale [4 6]. In this framework, some previously unidentified systematic effects have been recently evidenced. If not properly taken into account in the data analysis and the experiment-theory comparison, neglecting these effects may translate into significant systematic errors. In this contribution we briefly review the status of the experimental and theoretical knowledge in regard to such systematic effects, and the impact of the latter on the current limits to non-Newtonian gravitational forces. A complementary tool to extend our knowledge of quantum effects in the macroworld is available by considering peculiar quantum effects in strong external fields. In this framework we discuss the possibility to study observable effects due to the influence of curved space-time on the vacuum expectation value of the Higgs field. It turns out that the mass of the electron and, to a smaller extent, of baryons, should be changed in the presence of a strong gravitational field. This in turn gives rise to spectroscopic shifts in atomic spectroscopy of a novel nature with respect to the usual Doppler, gravitational, or cosmological shifts, also raising conceptual puzzles about the assignment of masses via the Higgs mechanism.

\section{CASIMIR FORCE EXPERIMENTS AND NON-NEWTONIAN LIMITS IN THE MICROMETER RANGE}

All experiments performed on Casimir forces between conducting bodies since the first attempts by Spaarnay [7] have faced the difficulty of dealing with a voltage present even when the two surfaces are shorted. This potential, called contact or residual potential, is thought to arise from the different Volta potential of the conductors used for the electrical connections, with typical values in the $1-100 \mathrm{mV}$ range. Apart from being superimposed to the expected Casimir force signal, its presence precludes the possibility to reach small gaps as it will cause, without taking proper precautions, earlier contact between the two surfaces. This is particularly relevant for experiments, such as the one using AFM cantilevers, involving resonators with low stiffness. It is then customary to compensate the contact potential using an external bias voltage kept constant in the entire range of explored distances. However, while performing electrostatic calibrations of an apparatus aimed at measuring the Casimir force in the cylinder-plane configuration, we have found evidence for a distance-dependent contact potential. Intrigued by this finding, we have studied the simpler sphere-plane configuration, since the latter is free from possible issues related to the parallelism and border effects potentially important in the cylinder-plane case. Even in the sphere-plane configuration, we have found a dependence of the contact potential upon distance, as well as an anomalous scaling of the electrostatic force with distance [8]. While this second anomaly does not seem of universal character [9], the dependence of the contact 
potential on distance has been confirmed by both reanalyzing former experiments (as discussed in [10]), and performing new experiments [9, 11]. The presence of a distance-dependent contact potential creates an electrostatic force unevenly compensated at the various explored distances, unless on-line [9] or off-line [10] compensation techniques are adopted.

The situation remains far from being settled, since there are also several experimental results interpreted as if there is no distance dependence of the contact potential, within the precision of the measurement [12, 13]. This issue is of the outmost importance to assess the best upper limits on Yukawa-like forces using molecular and Casimir forces [14, 15], as well as claims of validation of one specific model to include the finite conductivity and finite temperature correction [16]. Indeed, both the upper limits on Yukawa forces and the validation of thermal corrections rely on the two experimental apparata in which no systematic dependence of the contact potential on distance is observed (see [17] for details). The effect observed in the other experiments is of the order of $4-10 \mathrm{mV}$ in a range of distances of few $\mu \mathrm{m}$. This voltage should be compared with the equivalent voltage corresponding to the Casimir force at a given distance [18]. When this is done, a simple and universal formula, apart from a numerical factor, emerges for the equivalent voltage of the three geometries. As seen in Table I, the equivalent voltage of the Casimir force at $1 \mu \mathrm{m}$ is in the 10-20 $\mathrm{mV}$ range depending on the specific geometry, and this implies that the expected signal at $1 \mu \mathrm{m}$ for the Casimir force may be comparable, within one order of magnitude, to the difference between the signals coming from the contact potentials at the two extremes of the explored range of distances.

The current setting is clearly unsatisfactory to the purpose of providing reliable and accurate limits to nonNewtonian forces, regardless of the extent of the systematic effect. Indeed, even without carrying out extensive assessments of the precision of the experiments, we can identify three possible scenarios. If this previously unidentified systematic effect is larger than the quoted systematic error in the experiments used to give the best limits on Yukawa forces, then limits should be revised taking into account this systematic source of error. If it is instead smaller than the quoted systematic error in the same experiments, then one needs to understand whether these experiments, unable to evidence such an effect unlike all others, are actually suitable to provide the best limits to non-Newtonian gravitation. Finally, there is the possibility that specific environmental factors varying from experiment to experiment, such as the geometrical quality and chemical contamination of the surfaces, the radius of curvature of the sphere, the vacuum level, specific electrostatic setting around the apparatus, and temperature control for instance, may explain the different observations. Moreover, the same intrinsic geometry of the Casimir cavity seems to be relevant, as there is no evidence so far of the dependence of the contact potential on distance in the case of the parallel plate configuration [19 21], as we will report in a future publication. Evidently more experimental and phenomenological work in this direction will be required to provide a consistent framework.

A second issue only addressed quite recently is the validity of the proximity force approximation (PFA) [22] for Yukawa or, in general, volumetric forces. Since the PFA was conceived to deal with proximity forces, i.e. forces acting among entities in proximity of each other, it is not a priori understood what is their range of validity, and the level of accuracy, whenever PFA is applied to forces acting among entities in the bulk. A simplified form of PFA based on a virtual mapping between the actual sphere-plane configuration used in the experiments and an effective parallel plane configuration, used for instance in [16], has been recently shown to differ from the general expression for the PFA to be used in the case of volumetric forces [23]. This latter form has been in turn shown to coincide with the exact force between two bodies, provided one is an indefinite plane [24]. In the same paper, the sensitivity of the simplified PFA approximation to an unphysical parameter used in the PFA mapping between the sphere-plane and the parallel plane case, the thickness of the hypothetical slab corresponding to the sphere, denoted $D_{2}$ in 23.

\begin{tabular}{|l|c|c|c|}
\hline & Sphere-Plane & Cylinder-Plane & Parallel Planes \\
\hline \hline Casimir & $\left(\pi^{3} / 360\right) \hbar c\left(R / d^{3}\right)$ & $\left(\pi^{3} / 384 \sqrt{2}\right) \hbar c\left(L a^{1 / 2} / d^{7 / 2}\right)$ & $\left(\pi^{2} / 240\right) \hbar c\left(S / d^{4}\right)$ \\
\hline Coulomb & $\pi \epsilon_{0}(R / d) V^{2}$ & $\left(\pi \epsilon_{0} / 2 \sqrt{2}\right)\left(L a^{1 / 2} / d^{3 / 2}\right) V^{2}$ & $\left(\epsilon_{0} / 2\right)\left(S / d^{2}\right) V^{2}$ \\
\hline$V_{\text {Cas }}^{\mathrm{eq}}(\mathrm{d})$ & $\left(\pi^{2} / 360\right)^{1 / 2}\left(\hbar c / \epsilon_{0}\right)^{1 / 2} 1 / d$ & $\left(\pi^{2} / 120\right)^{1 / 2}\left(\hbar c / \epsilon_{0}\right)^{1 / 2} 1 / d$ & $\left(\pi^{2} / 192\right)^{1 / 2}\left(\hbar c / \epsilon_{0}\right)^{1 / 2} 1 / d$ \\
\hline$V_{\text {Cas }}^{\mathrm{eq}}(1 \mu \mathrm{m})$ & $9.85 \mathrm{mV}$ & $13.5 \mathrm{mV}$ & $17.1 \mathrm{mV}$ \\
\hline
\end{tabular}

TABLE I: Summary of relevant formulas for the ideal Casimir force and the Coulomb force in the cases of the sphere-plane, cylinder-plane, and parallel plane geometries. In the first row, the Casimir force is expressed by regrouping the various numerical factors and variables in a common fashion, with numerical values first, then the product $\hbar c$ expected in any Casimir force formula, then the geometrical dependence. The second row represents, in a similar arrangement, the Coulomb force exterted between the various surfaces. In the third row the equivalent Casimir voltage, i.e. the bias voltage required to simulate the Casimir force at a given distance $d$, is reported. In the last row the concrete value of the equivalent Casimir voltage is reported in the case of a typical gap distance of $1 \mu \mathrm{m}$. Notice the universality of the equivalent Casimir voltage formulas, with just different numerical factors giving rise to a difference of less than a factor two between the two extreme geometries. 
and [24], has been also studied. Taking into account the dependence on this parameter, limits derived through the simplified PFA become quickly unreliable as the Compton wavelength of the Yukawa force approaches the micrometer range, i.e. the upper range of distances in which precision Casimir force measurements have been performed. Then the validity of the PFA in the case of volumetric forces of Yukawa type is limited not only by the usual constraint of distance being much smaller than the radius of curvature of the sphere, $a \ll R$, but also by Yukawa range $\lambda \ll R, D_{2}$. This limits its conceptual validity to the case of nearly pointlike interactions and, above all, to a region of distances smaller than the one in which actual experiments are performed. This issue is not of practical concern for current experiments due to the dominance of the precision (of order $1.0 \%$ in the explored range of distances, see Fig. 10 in [16]) with respect to the estimated approximation in using the PFA formula for the Casimir force rather than its exact expression (of order $0.1 \%$ ). As the experimental precision will improve, for instance by developing low-temperature experiments in the submicrometer range, in analogy to the experiment described in [25] with attoNewton sensitivity, and if the sphere-plane PFA formula for the Casimir force will be replaced by its exact expression, the use of the exact Yukawa force will become increasingly crucial. The exact expression for the Yukawa force in the sphere-plane geometry, already available since a decade [26], has been recently used to improve the limits in the micrometer range using a torsional balance [27, 28].

\section{HIGGS SHIFTS IN ASTROPHYSICAL ENVIRONMENTS}

One of the most important predictions of the standard model of particle physics is the existence of the only fundamental scalar particle held responsible for the spontaneous symmetry breaking of the electroweak sector, providing mass to the intermediate vector bosons $W^{ \pm}$and $Z^{0}$ and to all fundamental fermionic matter fields. The Higgs particle is a critical milestone of the standard model, and its discovery and detailed study is the primary focus of research undergoing at high energy accelerators such as Fermilab and, in the close future, at the Large Hadron Collider at CERN. The Higgs particle, if giving mass to all the constituents of matter and provided that it satisfies the equivalence principle, should also play a crucial role in gravitational phenomena. In particular, the Higgs vacuum expectation value in a region of strongly curved space-time should differ from the one in flat space-time. This should give rise to different values for the mass of particles such as electrons and protons, and then to shifts of energy levels of their bound states of spectroscopic relevance. New wavelength shifts in the emission or absorption spectra are then predicted, which could be detected by proper subtraction of the usual Doppler, gravitational, and cosmological shifts.

Quantum field theory in curved space-time has been studied for decades both for non-interacting and interacting fields [29]. The Lagrangian density for an interacting scalar field with parameters $\mu$ and $\lambda$ in a generic curved space-time is written as:

$$
L=\frac{1}{2} g^{\mu \nu} \partial_{\mu} \phi \partial_{\nu} \phi-\frac{1}{2}\left(\mu^{2}+\xi R\right) \phi^{2}-\frac{\lambda}{4} \phi^{4} .
$$

In the presence of a curved space-time there is an added term to the Lagrangian density with respect to the case of a flat space-time, where $\xi$ measures the coupling between the Higgs field $\phi$ and the curvature scalar $R$. In the minimal coupling scenario, we should have $\xi=0$, which however is unnatural if we believe that the standard model at some energy will merge with gravitation, since it precludes any possible crosstalk between the two sectors. Moreover, it does not manifest proper renormalization group behavior, as outlined in [29] and, in a composite scalar model, in [30]. Alternatively, we consider as a working and/or reasonable hypothesis the other possibility of a conformal coupling, $\xi=1 / 6$. In the spontaneously broken phase, the Higgs field develops a vacuum expectation value obtained by minimizing the effective potential. In the flat space-time this yields a value $v_{0}=\sqrt{-\mu^{2} / \lambda}$, and the masses of the elementary particles are all directly proportional to $v$ via the Yukawa coefficients $y_{i}$ of the fermion-Higgs Lagrangian density term, $m_{i}=y_{i} v_{0} / \sqrt{2}$. In the presence of curved space-time, the effective coefficient of the Higgs field $\mu^{2} \mapsto \mu^{2}+\xi R$ and the vacuum expectation value of the Higgs field will become space-time dependent through the curvature scalar as:

$$
v=\sqrt{-\frac{\mu^{2}+\xi R}{\lambda}} \simeq v_{0}\left(1+\frac{\xi R}{2 \mu^{2}}\right),
$$

where the last expression holds for a weak curvature. Notice that the vacuum expectation value is increased by the presence of a curved space-time corresponding to $R>0$ in the minimal coupling scenario. In the case of elementary particles such as the electrons, provided that the Yukawa couplings are constants yet to be determined - as commonly believed, from algebraic/group theoretic arguments of an underlying fundamental theory incorporating the standard 
model - the mass $m_{e}$ will be simply changed proportionally to the Higgs vacuum expectation value, so that in the case of the electron $\delta m_{e}=y_{e}\left(v-v_{0}\right) / \sqrt{2} \simeq y_{e} \xi R v_{0} /\left(2^{3 / 2} \mu^{2}\right)=m_{e} \xi R /\left(2 \mu^{2}\right)$.

The situation for composite particles such as protons and neutrons is more involved. We assume that their masses are made of a flavor-dependent contribution proportional to the masses of the three valence quarks determined by the Higgs coupling, and a color-symmetrical term only dependent on the quark-quark and quark-gluon interaction, i.e. proportional to $\Lambda_{Q C D} \simeq 300 \mathrm{MeV}$. The latter dominates for lighter, relativistic quarks such as the up and down quarks constituting the valence component of protons and neutrons. Then, due to the universality of the QCD coupling constant for different flavors and for all gluons exchange, the QCD-related mass term will be the same for protons and neutrons, and therefore we will have:

$$
m_{p}=\left(2 y_{u}+y_{d}\right) v / \sqrt{2}+m_{Q C D}, \quad m_{n}=\left(y_{u}+2 y_{d}\right) v / \sqrt{2}+m_{Q C D} .
$$

For a generic atom of atomic number $Z$ and atomic mass $A$ we obtain:

$$
M(A, Z)=\frac{1}{\sqrt{2}}\left[y_{u}(Z+A)+y_{d}(2 A-Z)\right] v+A m_{Q C D}
$$

where we have neglected, to first approximation, the electron mass, the electron-nucleus binding energy and the nucleon binding energy. The purely QCD-dependent mass term should be independent on the curvature of spacetime, since otherwise the gluon could acquire a mass giving rise to the explicit breaking of the color symmetry. This is analogous to the case of the other unbroken symmetry of the standard model, $U(1)_{\text {em }}$ leading to the electromagnetic charge being conserved even in a generic curved space-time.

The possibility to detect Higgs shifts in atomic and molecular spectroscopy relies on the fact that electronic transitions depend primarily on the mass of the electron, whereas molecular transitions due to vibrational or rotational degrees of freedom depend upon the mass of the nuclei. While the electron mass is directly proportional to the Yukawa coupling, the mass of the nuclei is mainly due to the mass of its proton and neutron constituents, which in turn depends mainly on the color binding energy. We therefore expect that molecular transitions will not be affected by the Higgs shifts at leading order, unlike electronic transitions, not even in the most sensitive case of pyramidal molecules such as ammonia, for which tunneling provides enhanced sensitivity to changes in masses of the nuclei for the inversion lines. It is difficult to detect electronic, vibrational, and rotational transitions in the same region of space from the same species for a gas at thermal equilibrium, due to the very different energy scale difference required to produce these excitations. A comparative analysis of wavelength shifts from different species seems then necessary. This also enables to disentangle the putative Higgs shift contribution from the Doppler shift and the purely gravitational shift. The Doppler shift should be the same for molecules belonging to the same comoving cloud, while the wavelength shift expected from general relativity will act universally on all particles. Thus, unlike the Higgs shift, the latter will not discriminate between fundamental particles and interactions binding energies, i.e., between the electron mass and the main contribution to the proton mass due to QCD. A promising object to look for possible Higgs shifts is the Galactic center, where a compact object with an estimated mass of $M \simeq 2.6 \times 10^{6}$ solar masses, Sagittarius $\mathrm{A}^{*}$ [31 35], is present and supposed to be a black hole with a Schwarzschild radius of $R_{s}=2 G M / c^{2} \simeq 4 \times 10^{9} \mathrm{~m}$. Unfortunately, the curvature scalar is $R=0$ for a Schwarzschild black hole. Under the hypothesis that the Higgs field is coupled to another scalar invariant, for instance the Kretschmann invariant $K_{1}=R_{\mu \nu \rho \sigma} R^{\mu \nu \rho \sigma}$, then we have $\mu^{2} \mapsto \mu^{2}+\xi^{\prime} K_{1}^{1 / 2}$. For the Schwarzschild metric $K_{1}=12 R_{s}^{2} / r^{6}$, where $r$ is the distance from the center of the mass, and therefore we obtain $\mu^{2} \mapsto \mu^{2}\left(1+2 \sqrt{3} \xi^{\prime} R_{s} \lambda_{\mu}^{2} / r^{3}\right)$, where $\lambda_{\mu}=\hbar /(\mu c)$ is the Compton wavelength associated to the Higgs mass parameter $\mu$. Assuming a Higgs mass of $160 \mathrm{GeV}$, with $v=247 \mathrm{GeV}$, we obtain a Compton wavelength $\lambda_{\mu} \simeq 2 \times 10^{-18} \mathrm{~m}$. Even if we consider the innermost stable orbit around a solar mass black hole, at $r=3 R_{s}$, we obtain $K_{1}^{1 / 2}=(4 / 243)^{1 / 2} R_{s}^{-2}=1.5 \times 10^{-8} \mathrm{~m}^{-2}$. Therefore the product of the squared Compton wavelength and the Kretschmann term is $\lambda_{\mu}^{2} K_{1}^{1 / 2} \simeq 6 \times 10^{-44}$, leading to Higgs shifts very far from observational reach. The main issue here is the mismatch in the lengthscales $\lambda_{\mu}$ and $K_{1}^{-1 / 4}$ which could be reduced if mini black holes are considered. It should be also noticed that the lack of detailed knowledge of compact objects cannot rule out their possible description in terms of naked singularities, providing effective curvatures better matched to $\lambda_{\mu}$, although the very existence of naked singularities is a hotly debated theme in general relativity [36]. Upper bounds on the existence of Higgs shifts from dedicated surveys can be anyway useful to constraint proposals in which the Higgs boson is supposed to be responsible for inflation via anomalously large coupling to the metric, as proposed in [37, 38].

As benchmarks from the observational viewpoint, with a 1 pc resolution survey it is now possible to obtain spectra of atoms or molecules at a distance of $r \simeq 2 \times 10^{16} \mathrm{~m}$ from the Galactic center, and recent surveys of ammonia [39, 40] have a spectral sensitivity corresponding to a Doppler shift of about $2-3 \mathrm{~km} / \mathrm{s}$, i.e. $\delta \nu / \nu \simeq 10^{-5}$. In the case of atomic 
hydrogen spectroscopy, the spectral lines depend directly on the reduced mass $\mu_{e p}=m_{e} m_{p} /\left(m_{e}+m_{p}\right)$ and ultimately, due to the large mass ratio $m_{p} / m_{e}$, on the electron mass. Observation of atomic lines from the Galactic center is difficult due to the strong absorption at optical wavelengths, and therefore one should focus on the high-precision monitoring of the 21-cm neutral hydrogen line, still dependent on the electron to proton mass ratio. This implies measuring the 21-cm neutral hydrogen line from interstellar clouds near the Galactic Center, or from stars with highly eccentric orbits. In the latter case, clear signatures might be available by looking at the temporal variability of the $21 \mathrm{~cm}$ hyperfine line along the star orbit.

Finally, we want to point out that in the standard model the masses of fundamental particles have a different treatment with respect to the mass coming from interaction among themselves. If the equivalence principle holds, the gravitational mass of the electrons constituting a test body will change if the Higgs field is coupled to curvature, while the nucleons will continue to keep, to leading order, the usual gravitational charge. This is in striking contrast to the case of general relativity where all sources of energy contribute without any distinctive feature to the emergence of space-time, originating at least an unappealing contrast in the way masses are considered in Higgs physics and gravitation. Stability issues of back-reaction on the metric may also arise depending on the relative signs of $\xi$ and $R$, potentially originating catastrophic growths of the local curvature and of the mass of the test particles.

\section{CONCLUSIONS}

We have briefly discussed two critical issues in the interplay between macroscopic quantum vacuum and microscopic gravitation, namely the need for a more careful analysis of systematic effects in Casimir force measurements to improve our knowledge of possible non-Newtonian forces of gravitational origin, and the influence of strong spacetime curvature on the generation of mass generation via the Higgs field. By smearing out the two traditional regions of applicability of quantum physics and gravitation, the microworld and the macroworld respectively, one could gain important insights on their mutual compatibility, as already attempted in [41 48] for the relationship between vacuum energy and curved space-time, and in [49, 50] concerning the validity of the equivalence principle for macroscopically distinguishable quantum states. All this should be considered part of a program aimed at narrowing the gap between the physics of quantum vacuum and the standard model of elementary particle physics and its proposed extensions, as recently outlined in [51].

\section{Acknowledgments}

I would like to thank the QFEXT09 organizers for the kind invitation to such a stimulating and successful meeting. I also acknowledge partial support from the Julian Schwinger Foundation through grant JSF 08070000 on Astrophysics of Quantum Vacuum.

\section{REFERENCES}

[1] M. Bordag, U. Mohideen, and V. M. Mostepanenko, Phys. Rep. 353, 1 (2001).

[2] S. K. Lamoreaux, Rep. Progr. Phys. 68, 201 (2005).

[3] For a different viewpoint see: C. R. Hagen, Eur. Phys. J. C 19, 677 (2001).

[4] S. Dimopoulos and G. F. Giudice, Phys. Lett. B 379, 105 (1996).

[5] Y. Fujii, Nature 234, 5 (1971); Ann. Phys. 69, 494 (1972); Phys. Rev. D 9874 (1974); Int. J. Mod. Phys. A 6, 3505 (1991).

[6] E. Fischbach and C. L. Talmadge, The Search for Non-Newtonian Gravity (AIP/Springer-Verlag, New York, 1999).

[7] M. J. Sparnaay, Physica 24, 751 (1948).

[8] W. J. Kim, M. Brown-Hayes, D. A. R. Dalvit, J. H. Brownell, and R. Onofrio, Phys. Rev. A 78, 020101(R) (2008).

[9] S. de Man, K. Heeck, and D. Iannuzzi, Phys. Rev. A 79, 024102 (2009).

[10] W. J. Kim, M. Brown-Hayes, D. A. R. Dalvit, J. H. Brownell, and R. Onofrio, J. Phys. Conf. Ser. 161, 012104 (2009).

[11] W. J. Kim, A. O. Sushkov, D. A. R. Dalvit, and S. K. Lamoreaux, Phys. Rev. Lett. 103, 060401 (2009).

[12] H.-C. Chiu, C.-C. Chang, R. Castillo-Garza, F. Chen, and U. Mohideen, J. Phys. A 41, 164022 (2008).

[13] R. S. Decca, E. Fischbach, G. L. Klimchitskaya, D. E. Krause, D. López, U. Mohideen, and V. M. Mostepanenko, Phys. Rev. A 79, 026101 (2009). 
[14] V. A. Kuzmin, I. I. Tkachev, and M. E. Shaposhnikov, Pis'ma Zh. Eksp. Teor. Fiz. 36, 49 (1982) [JETP Lett. 36, 59 (1982)].

[15] V. M. Mostepanenko and I. Yu. Sokolov, Phys. Lett. A 125, 405 (1987).

[16] R. S. Decca, D. López, E. Fischbach, G. L. Klimchitskaya, D. E. Krause, and V. M. Mostepanenko, Ann. Phys. (N.Y.) 318, 37 (2005).

[17] G. L. Klimchitskaya, U. Mohideen, and V. M. Mostepanenko, Rev. Mod. Phys. 81, 1827 (2009).

[18] R. Onofrio and G. Carugno, Phys. Lett. A 198, 365 (2005).

[19] G. Bressi, G. Carugno, A. Galvani, R. Onofrio, G. Ruoso, and F. Veronese, Class. Quantum Grav. 18, 3943 (2001).

[20] G. Bressi, G. Carugno, R. Onofrio, and G. Ruoso, Phys. Rev. Lett. 88, 041804 (2002).

[21] P. Antonini, G. Bimonte, G. Bressi, G. Carugno, G. Galeazzi, G. Messineo, and G. Ruoso, J. Phys. Conf. Ser. 161, 012006 (2009).

[22] B. V. Derjaguin and I.I. Abrikosova, Sov. Phys. JETP 3, 819 (1957).

[23] R. S. Decca, E. Fischbach, G. L. Klimchitskaya, D. E. Krause, D. López, and V. M. Mostepanenko, Phys. Rev. D 79, 124021 (2009).

[24] D. A. R. Dalvit and R. Onofrio, Phys. Rev. D 80, 064025 (2009).

[25] S. J. Mullin, A. A. Geraci, D. M. Weld, J. Chiaverini, S. Holmes, and A. Kapitulnik, Phys. Rev. D 72, 122001 (2005).

[26] M. Bordag, B. Geyer, G. L. Klimchitskaya, and V. M. Mostepanenko, Phys. Rev. D 58, 075003 (1998).

[27] M. Masuda, M. Sakaki, and A. Araya, Class. Quantum Grav. 24, 3965 (2007).

[28] M. Masuda and M. Sasaki, Phys. Rev. Lett. 102, 171101 (2009).

[29] N. D. Birrell and P. C. W. Davies, Quantum Fields in Curved Space, (Cambridge University Press, 1982).

[30] C. T. Hill and D. S. Salopek, Ann. Phys. (N.Y.) 213, 21 (1992).

[31] M. Morris and E. Serabyn, Annu. Rev. Astron. Astrophys. 34, 645 (1996).

[32] A. Eckart and R. Genzel, Mon. Not. R. Astron. Soc. 284, 576 (1997).

[33] R. Genzel, A. Eckart, T. Ott, and F. Eisenhauer, Mon. Not. R. Astron. Soc. 291, 219 (1997).

[34] A. M. Ghez, B. L. Klein, M. Morris, and E. E. Blecklin, Astrophys. J. 509, 678 (1998).

[35] M. J. Reid, A. C. S. Readhead, R. C. Vermeulen, and R. N. Treuhaft, Astrophys. J. 524, 816 (1999).

[36] P. S. Joshi, Pramana 55, 529 (2000); 69, 119 (2007).

[37] F. Bezrukov and M. Shaploshnikov, Phys. Lett. B 659, 703 (2008).

[38] F. Bezrukov, A. Magnin, and M. Shaploshnikov, Phys. Lett. B 675, 88 (2009).

[39] C. Henkel, N. Jethava, A. Kraus, K. M. Menten, C. L. Carilli, M. Grasshoff,D. Lubowich, and M. J. Reid, Astron. Astrophys. 440, 893 (2005).

[40] T. L. Wilson, C. Henkel, and S. Höttemeister, Astron. Astrophys. 460, 533 (2006).

[41] M. Karim, Phys. Lett. A 171, 7 (1992).

[42] M. Karim, A. H. Bokhari, and B. J. Ahmedov, Class. Quantum Grav. 17, 2459 (2000).

[43] E. Calloni, L. Di Fiore, G. Esposito, L. Milano, and L. Rosa, Phys. Lett. A 297, 328 (2002).

[44] F. Sorge, Class. Quantum Grav. 22, 5109 (2005).

[45] S. A. Fulling, K. A. Milton, P. Parashar, A. Romeo, K. V. Shajesh, and J. Wagner, Phys. Rev. D 76, 025004 (2007).

[46] K. A. Milton, P. Parashar, K. V. Shajesh, and J. Wagner, J. Phys. A 40, 10395 (2007).

[47] K. V. Shajeah, K. A. Milton, P. Parashar, and J. A. Wagner, J. Phys. A 41, 164058 (2008).

[48] K. A. Milton, S. A. Fulling, P. Parashar, A. Romeo, K. V. Shajesh, and J. A. Wagner, J. Phys. A 41, 164052 (2008).

[49] L. Viola and R. Onofrio, Phys. Rev. D 55, 455 (1997).

[50] R. Onofrio and L. Viola, Mod. Phys. Lett. A 12, 1411 (1997).

[51] H. Gies, J. Phys. A: Math. Theor. 41, 164039 (2008). 